\title{
Dentist Role in Oral Treatment Before Head Neck Cancer Therapy
}

\author{
Rahmatullah Irfani ${ }^{1}$, Anandina Irmagita Soegyanto ${ }^{2 *}$ \\ ${ }^{1}$ Resident of Oral Medicine Residency Program, Faculty of Dentistry, Universitas Indonesia, Jakarta 10430, \\ Indonesia \\ 2. Oral Medicine Department, Faculty of Dentistry, Universitas Indonesia, Jakarta 10430 Indonesia \\ *E-mail: a.irmagita@gmail.com
}

\begin{abstract}
Background: Various intra-oral conditions can be found in patient prior the cancer treatment that might include lesions associated with decreased immunity, such as viral infections and fungal infections. Dentist has a significant role to prepare the oral cavity to be in the best possible condition, in order to minimize oral disturbance and complaints of cancer patients.
\end{abstract}

Objective: The purpose of this article is to review the importance of intra-oral treatment prior cancer therapy in patients with head and neck cancer.

Case Report: A 35-years-old male patient was referred from the ENT Department, Cipto Mangunkusumo Hospital with suspected Nasopharyngeal Carcinoma for investigating the intra oral focal infection sources. Intra oral examination revealed various focal infection sources and pseudomembranous candidiasis. The candidiasis was completely healed after antifungal medication.

Conclusion: Dentists' role in preparing the oral condition prior cancer treatment is very important to reduce further oral complication. All oral lesions including fungal infection should be treated before the commencement of cancer treatment.

Keywords: cancer therapy, candidiasis, oral treatment

\section{Introduction}

Cancer is a condition in which the uncontrolled of tissue growth that result from an imbalance between cell division and apoptosis. ${ }^{1}$ Cancer can be experienced by nearly every complex multicellular organism. It involves in the process of disruption in proliferation, differentiation, senescence and apoptosis. ${ }^{2}$ Etiology of cancer vary, but a wide range of risk factors can be determined as of the food, the environment, tobacco and excessive alcohol consumption. Head and neck cancer (HANC) states the variation of tumors existing in the aero-digestive tract of the head and neck. ${ }^{3}$

Pretreatment oral evaluation is suggested for patients with cancer before the initiation of therapy. The aims of the evaluation are to eliminated oral disease that may get worse during cancer therapy, provide a baseline for assessment and sequelae monitoring of cancer therapy damage, detect metastatic lesions, and finally, is to minimize oral discomfort during cancer therapy. ${ }^{1}$

Dentist has an important role in the management of patient with cancer. The primary role is in early recognition of the disease. Moreover, the dentist is responsible for all fitness of oral cavity before, during and after cancer treatment is executed. A good oral health condition is very closely related with overall body, as it's important role in the function of digestive. Healthy oral condition may also prevent the onset of psychological problems that may arise as a result of oral complications related to cancer therapy.

\section{Objective}

In this paper, we report a case of nasopharyngeal carcinoma patient who suffered from pseudomembranous candidiasis. We also discuss the intra-oral conditions can be found in patients with head and neck cancer and the role of dentists in the intra-oral treatment before the cancer therapy.

\section{Case Report}

A 35-years-old male patient was referred from Ear Nose Throat (ENT) Department with Nasopharyngeal carcinoma for investigating the intra oral focal infection 
sources. There was a history of smoking since childhood and a history of drinking alcohol since 5 years ago. There were complaints numbness on the left cheek, somewhat diminished hearing, difficulty in swallowing and the clogged nose. In addition, the left eye looked swollen and the patient also admitted to have impair vision. The patient consumed $0.5 \mathrm{mg}$ of oral corticosteroid 4 times daily which was given by neurology. Pathology examination of the cancer was resulting in the conclusion of Nasopharyngeal Carcinoma, not keratinized, undifferentiated, type A, with medium degree of malignancy.

Intra oral examination revealed poor oral hygiene condition, with gingival inflammation, tooth remnants on $28,37,36$, dentinal caries on $26,27,38,46$, and some white patches with erythematous base in almost all oral mucosal surface (Figure 1). The patient was diagnosed to have Pseudomembranous candidiasis, with various focal infection sources such as chronic gingivitis and tooth radix on 28, 37, 36 and pulp hyperemia on $26,27,38,46$.

At the first visit, the patient was educated and instructed about the importance oral health and hygiene maintenance, especially since he would undergo cancer therapy. In order to eliminate all focal infection sources and to improve his oral condition, the patient was recommended to perform scaling and root planning, residual root extraction and also cavity filling, but these dental treatments could not be performed yet since the patient did not have complete blood count examination result yet. For the treatment of pseudomembranous candidiasis, Nystatin oral suspension was prescribed at a dose of $1 \mathrm{ml} 4$ times a day, to be swished and swallowed.

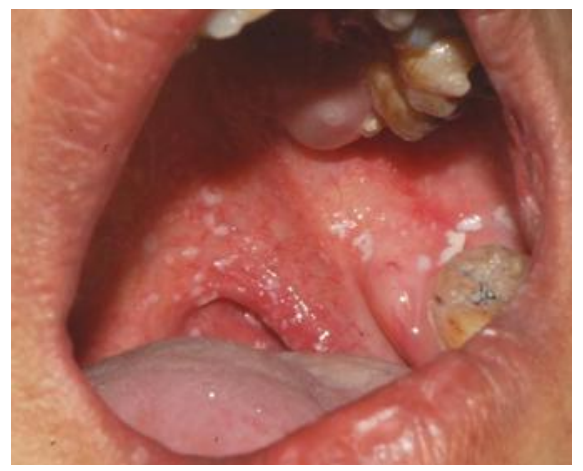

Figure 1. White Patches on Oral Mucosa

On the follow up visit, a week after the first visit, the patient felt more comfortable about his oral condition. On examination, the white patches of oral mucosa have reduced significantly. To completely remove the Candida infection, he was instructed to finish all nystatin oral solution prescribed previously. Then the patient was planned to continue the intra oral treatment to remove focal infection source by scaling and residual tooth extractions. When all the dental treatment was completed, the patient could return to the ENT department and continued his cancer treatment. Patient was also reminded to always maintain his oral hygiene during his cancer treatment, and should return immediately to dental clinic, should he experience any oral discomfort during and after his cancer treatment.

\section{Discussion}

In this case, the nasopharyngeal carcinoma patient was referred to dental clinic before undergoing his cancer treatment. Prior to cancer therapy, all condition in patients with nasopharyngeal carcinoma should be evaluated, including the oral conditions. The evaluation includes examination of caries, oral mucosa, and other dental diagnosis that could potentially become a source of focal infection. The focus of the pre-treatment examination is to determine the oral health status and the things that need to be done prior to the oral cancer therapy. These actions are done in order to prevent complications that may arise during and after cancer treatment, or at least to reduce the possibility of complication severity. ${ }^{4}$

During our patient intra oral examination, there were white patches with erythematous base that almost covered all oral mucosal surfaces, and diagnosed as pseudomembranous candidiasis. The appearance of lesion was very distinctive so that it did not require any further supportive examination. ${ }^{5}$ This fungal infection is common to happen in patient with carcinoma. The prevalence of oral fungal colonization in cancer patients was $48.2 \%$ before treatment, $72.2 \%$ during treatment, and $70.1 \%$ after treatment. ${ }^{6}$ A significant increase for intra oral fungal infection also can be associated with radiotherapy and chemotherapy in HANC patient. In this case, the patient was also given corticosteroid from other department for a reason that was not identified by the dentist. Corticosteroid treatment might also predispose to fungal infection. $^{5}$

For the treatment of oral candidiasis, anti-fungal drug application can be considered. ${ }^{7}$ And In our case, the use of nystatin oral suspension brought a good result. Nystatin is a topical antifungal agent that work effectively for fungal infections. $^{8}$

The oral condition in the patient was not in good shape. Various focal infection sources can be found in his mouth, including gingival inflammation, tooth remnants and dental caries. Those conditions need to be restored prior the start of his cancer treatment. Oral pre-treatment of cancer therapies need to be done in order to prevent excessive pain during therapy, lowers the risk of infection and specially to prepare oral conditions in the best condition. Some possible complications that would be suffered by patients undergoing cancer therapy might include: mucositis, infections, xerostomia, changes in taste ability, and decreased ability of mastication. These complications can lead to a decrease in nutritional status of patients that may disrupt the success of cancer treatment. ${ }^{6,9,10}$ Eventually, these oral complications will also affect the total cost of the patient during cancer therapy. ${ }^{6}$ Therefore, it is very important to reduce the risk of oral complications during and after cancer therapy by performing the proper oral pretreatment. 
In patients who suffered head and neck cancer, oral mucosal changes are frequently seen. It may be associated with the proximity location between the cancer and oral cavity, and related to decrease in the immune status of patients. ${ }^{11}$ These changes can also be caused by immunosuppressive condition (influence of drugs or disease), hypo salivation (influenced by drugs, disease or radiotherapy), damage to mucosal tissue of the mouth or mucositis (chemotherapy or radiotherapy induced). ${ }^{8}$ In addition, other more severe complications include radiation caries, trismus and osteoradionecrosis. ${ }^{6,10}$

This case report shows the importance of dental examination prior head and neck cancer therapy. As dentist is a health practitioner who has high responsibility in oral health care, that include the head and neck cancer patients. There are three stages that should become the focus of the dentist in cancer patient, which are prior, during and after the cancer therapy. ${ }^{12}$

The first stage is prior to cancer therapy. At this stage the condition of intra oral patients were evaluated to be prepared prior to cancer therapy. The required accuracy and appropriate decision making role in determining the focus of infection elimination action plan such as the handling of caries, periodontal treatment tooth extraction with poor prognosis. This action will eradicate possible infection sources that may disturb patient's general health during cancer treatment. ${ }^{13}$ It must be ensured that the intra-oral condition is free from infection. The patient in this case showed the presence of dental infection and candida infection in oral mucosa. These are the infections focused to be eliminated by the dentist.

The second stage is during cancer therapy, when dentist may find patients with treatment complication that needs to be treated. During chemotherapy and radiotherapy, patients could experience various intra oral complications. Infections, oral ulcerations, dry mouth, and bleeding are the most common complication experienced by them. These complications may vary, depends on the type of therapy, bone marrow status and other toxicities, and on the acuteness and severity of the complications ${ }^{4}$. During this stage, particularly in patients with advanced-stage malignancies, poorer oral hygiene is also common to find. These conditions should be informed to the patient and anticipated by the dentist. In this case, after the completion of dental treatment prior cancer therapy, the patient was encouraged to maintain the oral condition and should return if any oral complain exist. The dentist must educate the patient on the daily oral prophylaxis procedure and the side effect of therapy that might happen during the cancer treatment. ${ }^{9}$

The third stage is after the cancer therapy. Some chronic effects of therapy can develop and relapse in patients. HANC patient who undergo head and neck radiotherapy might experience xerostomia due to radiation effect toward salivary glands. Should the damage continue to the gland parenchyma, these complications might last a lifetime ${ }^{10}$. The condition could have an impact to the oral condition of the patient that eventually affect the patient's quality of life. Dentist has a role in the maintenance of intra-oral condition after the completion of the cancer therapy. It is important for dental professionals to work in a team, together with other specialties.

Here is shown that dentists have a vital role, which should not be marginalized, in treating patients with cancer, particularly the HANC patient. The most important of dentist role begins before cancer treatment, but follow-ups should also be done during and after cancer treatment. These actions will eventually support the success of cancer therapy.

\section{Conclusions}

Dentists have a very important role in performing dental examination and treatments for patient who would undergo cancer treatments, as various oral conditions that can influence treatment successful might be seen. Ideally, dentists continue to be involved in all stages of cancer therapy, to ensure intra-oral condition of the patient is in good condition for intra oral health is associated with the patient's general health.

\section{Acknowledgment}

The authors would like to acknowledge drg. Afi Savitri Sarsito Sp.PM for assisting the patients' clinical examinations.

\section{References}

1. V Singh, S Malik. Oral Care Of Patients Undergoing Chemotherapy And Radiotherapy: A Review Of Clinical Approach. The Internet Journal of Radiology. 2006; 6:1.

2. Meurman JH, Bascones-Martinez A. Are Oral and Dental Disease Linked to Cancer? Oral Diseases (2011) 17, 779784.

3. World Health Organization. International Statistical classification of disease and relative health problems. (10th ed.). Geneva: WHO; 2010. Available from www.who.int/classifications/icd/ICD10

4. Gilliam K, Oral Health Maintenance in Head and Neck Cancer Patients. PennWell Publication. 2014.63-76. Available from rdhmag.com

5. Iriyama $\mathrm{N}$, et al, Efficacy and safety of antifungal prophylaxis with oral itraconazole solution among patients receiving corticosteroids : who should be given prophylaxis. J of Infection and Chemotherapy $2011 ; 17: 744-9$.

6. Lalla RV,et al, A Systematic Review of Oral Fungal Infection in Patients Receiving Cancer Therapy. Support Care Cancer. 2010 August; 18(8):985-92.

7. Lopez BC, Esteve CG, Perez GS. Dental Treatment Considerations in the Chemotherapy Patient. J Clin Exp Dent. 2011;3(1):e31-42.

8. Rolim AEH, Da Costa LJ, Ramalho LMP Impact of Radiotherapy on the orofacial region and management of related conditions. Radiol Bras. 2011 Nov/Dez;44(6):38895.

9. Beech N, Robinson S, Porceddu S, Batstone M. Dental Management of Patients Irradiated for Head and Neck Cancer. Australian Dental Journal 2014; 59:20-8.

10. Migliorati CA, Seneda LM, Burton EL. Oral Complication of Cancer Therapy: A Summary Guide for The Clinician. Journal of The Tennessee Dental Association. 2014; 24-32.

11. Genden EM, Contemporary management of cancer of the oral cavity. Eur Arch Otorhinolaryngol (2010) 267:100117. 
12. Walsh LJ. Clinical assessment and management of the oral environment in the oncology patient. Australian Dental Journal 2010; 55:(1 Suppl): 66-77.

13. Khrisnan V. Systemic Affliction of Oral Focal Sepsis. J Indian Aca Oral Med Radiol 2012;24(2): 137-141. 